\title{
On the role of initial electronic coherence in molecular dissociation induced by an attosecond pulse
}

\author{
Lukas Medišauskas* \\ Department of Physics, Imperial College London, \\ South Kensington Campus, SW7 $2 A Z$ London, United Kingdom and \\ Max-Born-Institut, Max-Born Strasse 2A, D-12489 Berlin, Germany \\ Serguei Patchkovskii, Alex Harvey, Danilo S. Brambila, Christian \\ Neidel, Jesse Klei, Arnaud Rouzée, and Marc J.J. Vrakking \\ Max-Born-Institut, Max-Born Strasse 2A, D-12489 Berlin, Germany \\ Misha Yu. Ivanov \\ Max-Born-Institut, Max-Born Strasse 2A, D-12489 Berlin, Germany \\ Department of Physics, Humboldt University, \\ Newtonstr. 15, D-12489 Berlin, Germany and \\ Department of Physics, Imperial College London, \\ South Kensington Campus, SW7 $2 A Z$ London, United Kingdom
}

(Dated: September 21, 2015)

\begin{abstract}
We investigate the influence of the attosecond electron dynamics of photoionization on the femtosecond fragmentation of the molecular ion left behind. We consider the dissociative photoionization dynamics of the $\mathrm{N}_{2}$ molecule, induced by an attosecond extreme-ultraviolet (XUV) pulse in the presence of a moderately strong infrared (IR) laser field. We show that the kinetic energy spectrum of $\mathrm{N}^{+}$fragments depends on (i) the phases between the different electronic states of $\mathrm{N}_{2}^{+}$ established by the photoionization process and (ii) phases associated with the vibrational dynamics in the dissociating molecular ion. We show that the phase acquired during the photoionization can be obtained from the dependence of the $\mathrm{N}^{+}$ion kinetic energy release (KER) spectra on the time delay between the XUV and IR pulses.
\end{abstract}

\footnotetext{
* lukas.medisauskas@imperial.ac.uk
} 
Following the first few femtoseconds of a chemical reaction is an exciting opportunity created by the advent of sub-femtosecond light sources [1]. While the nuclei in a molecule move on tens to hundreds of femtoseconds timescale, sub-femtosecond timescales are pertinent to electronic motion - the creation and evolution of coherence between different electronic states. If the reaction is initiated by ultrafast ionization, how does the coherence between the ion and the continuum electron manifest?

We address this question theoretically by considering an attosecond pump-IR probe experiment. Using the $\mathrm{N}_{2}$ molecule as an example, we show that the kinetic energy release (KER) spectra of fragment ions, resulting from two-color dissociative ionization, are sensitive to the relative photoionization phases in different photoionization channels. Thus we reveal the essential role played by quantum entanglement between the molecular ion and the XUV-liberated photoelectron in the formation of the KER spectra. The presented mechanism will arise in any pump-probe experiment that involves an isolated attosecond pump pulse (or a train of attosecond pulses) and an IR probe pulse, phase-locked to the XUV pump.

The influence of coherent electronic dynamics on longer-term nuclear dynamics has been a subject of intense discussions, starting with the pioneering works [2-4] stimulated by the idea of charge-directed reactivity $[5,6]$. The role of attosecond electronic dynamics was first illustrated in an experiment [7] that demonstrated control of electron localization during molecular dissociation. Theoretically, such control was first studied for $\mathrm{H}_{2}$ [8] and $\mathrm{HCl}[9,10]$. Extending the work reported in [7], sub-fs control of electron localization and molecular fragmentation was observed in two-color XUV+IR experiments, demonstrating a sensitivity to the time-delay between the XUV pump and the oscillations of the IR probe electric field (see e.g. Ref. [11-13]). Contrary to these studies, here we focus on the interplay between the vibrational and photoionization time-scales and reveal the key role that the departing photoelectron plays in determining the outcome of photodissociation.

To investigate the correlated photoelectron-ion dynamics we express the full asymptotic wavefunction of the system after ionization by an XUV pulse in the close-coupling form as:

$$
|\Psi(t)\rangle=\sum_{i}\left|\phi_{i}(t)\right\rangle\left|\Psi_{i}(t)\right\rangle .
$$

Here $\left|\Psi_{i}(t)\right\rangle$ are the vibronic states of the ion that describe coupled electronic and nuclear dynamics associated with ionization channel $i$. Within the Born-Oppenheimer approximation 
the latter can be described in terms of vibrational wavepackets $\chi(R, t)$ moving on electronic potential curves of the ion. With each of these vibronic states comes a continuum electron wavepacket $\left|\phi_{i}(t)\right\rangle=\int \mathrm{d} E a_{i}^{E}(t)\left|\phi^{E}\right\rangle$, correlated to the vibronic wavepacket in channel $i$ in energy, momentum and symmetry. The $\left|\phi^{E}\right\rangle$ are asymptotic continuum states describing an electron with final energy $E$ and channel-specific complex amplitudes $a_{i}^{E}(t)$ (see appendix A for a more extended discussion).

Consider a measurement that resolves the vibrational continuum states of the molecular ion but integrates over the states of the photoelectron. This situation applies when only the fragment ion KER spectra are measured. One should in general specify a measurement basis $|\chi\rangle$ for detecting the KER and basis $|n\rangle$ for detecting the electronic eigenstates of the ionic fragments. This basis does not necessarily connect adiabatically to the electronic states initially populated by photoionization in channel $i$. This allows one to include processes such as autoionization and IR-induced transitions from an initially populated electronic state in channel $i$ towards a target eigenstate $|n\rangle$. Furthermore, it may not be possible to distinguish the initial electronic state after ionization in channel $i$, when several initial states lead to the same target eigenstate $|n\rangle$.

The probability to detect the ion in an electronic state $|n\rangle$ and vibrational state $|\chi\rangle$, integrated over all final states $|\phi\rangle$ of the continuum electron yields

$$
\begin{aligned}
& W_{n}(\chi, t)=\int \mathrm{d} \phi \mid\left\langle n \chi|\langle\phi \mid \Psi(t)\rangle|^{2}=\sum_{i}\left|\left\langle n \chi \mid \Psi_{i}(t)\right\rangle\right|^{2}\right. \\
& +\sum_{i \neq j}\left\langle\Psi_{j}(t) \mid n \chi\right\rangle\left\langle n \chi \mid \Psi_{i}(t)\right\rangle \times\left\langle\phi_{j}(t) \mid \phi_{i}(t)\right\rangle .
\end{aligned}
$$

Electronic coherence between the $i$ and $j$ channels created by photoionization appears in the second term on the right hand side of Eq. 2. This term must be non-zero for the coherence to affect the KER spectra. Therefore, wavepackets initially created in different ionization channels $i$ and $j$ must contribute to the same final electronic eigenstate $|n\rangle$ and the overlap $\left\langle\phi_{j}(t) \mid \phi_{i}(t)\right\rangle=\int \mathrm{d} E\left(a_{j}^{E}\right)^{*} a_{i}^{E}$ of the photo-electron wavepackets $\phi_{i, j}$ must be significant.

Because the continuum photo-electron wavepackets $\left|\phi_{i}(t)\right\rangle$ are correlated to different ionic states, they acquire different phases during photoionization. This phase difference affects the interference and thus the vibrationally resolved dissociation spectra in Eq. 2. Hence, manipulating the phase and magnitude of the initial electronic coherence created by at- 
tosecond ionization potentially changes the outcome of vibrational dynamics hundreds of femtoseconds later.

Theoretical and experimental studies showed that the amount of electronic coherence created after photoionization by strong IR fields and short XUV pulses can be significant, this is clearly observed in $[11,14,15]$, and can be controlled by the choice of laser parameters [16]. However, its role in chemical reactivity (i.e. molecular dissociation) and hole migration $[3,4,15]$ has so far not been addressed.

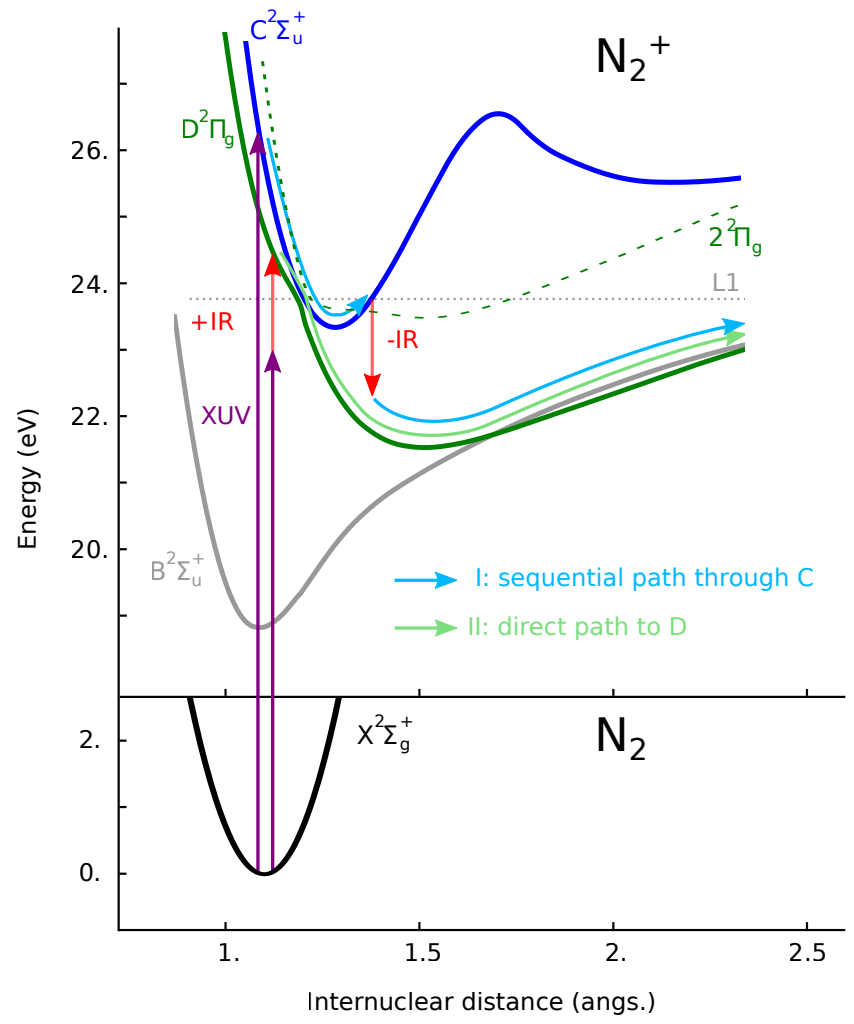

FIG. 1. Sequential XUV-IR (I) and direct XUV+IR (II) dissociation pathways leading to low energy $\mathrm{N}^{+}$fragments and the relevant potential energy curves (see text for further explanation). $2^{2} \Pi_{g}$ is the upper adiabatic branch of the $\mathrm{D}^{2} \Pi_{g}$ state, which for simplicity is not included in the calculation. The horizontal dotted line indicates the dissociation limit. The bandwidth of a 500 as (FWHM) pulse spans an energy range of $\sim 7 \mathrm{eV}$.

Here we theoretically demonstrate the importance of the initial electronic coherence created by ionization for the nuclear motion in the molecular ion. We use the example of dissociative ionization of $\mathrm{N}_{2}$ exposed to a combined XUV and IR laser field, with parameters similar to those in $[11,12,17]$. The IR pulse is $30 \mathrm{fs}$ FWHM, centred at $\lambda=800$ 
$\mathrm{nm}$, with a peak intensity of $10^{13} \mathrm{~W} / \mathrm{cm}^{2}$ and polarized perpendicular to the molecular axis.

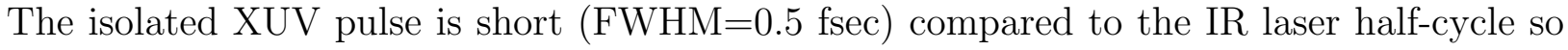
that photoionization is adequately described by a vertical Franck-Condon transition onto the cationic electronic states. The XUV carrier frequency $\Omega \simeq 25 \mathrm{eV}$ leads predominantly to the formation of the $\mathrm{X}^{2} \Sigma_{g}^{+}, \mathrm{A}^{2} \Pi_{u}$, and $\mathrm{B}^{2} \Sigma_{u}^{+}$bound ionic states, and low-lying vibrational levels of the $\mathrm{C}^{2} \Sigma_{u}^{+}$state $[18,19]$. The latter predissociate into the $\mathrm{B}^{2} \Sigma_{u}^{+}$state on nanosecond timescales, leading to a well known vibrational structure [20] in the low energy $\mathrm{N}^{+}$KER which is independent of XUV-IR delay.

The IR field creates additional two-photon $\mathrm{XUV} \pm \mathrm{IR}$ pathways towards fragmentation moving along the shallow $\mathrm{D}^{2} \Pi_{g}$ state potential energy curve (PEC) leading to $\mathrm{L}_{1}$ dissociation limit and producing low kinetic energy fragments (Figure 1). The first pathway, labelled XUV-IR (I), describes one-photon XUV ionization into the long-lived $\mathrm{C}^{2} \Sigma_{u}^{+}$state, followed by IR-induced resonant de-excitation onto the $\mathrm{D}^{2} \Pi_{g}$ state. The de-excitation probability is significant only well after the XUV pulse, when the vibrational wavepacket that is formed on the $\mathrm{C}^{2} \Sigma_{u}^{+}$state has moved to the outer turning point of the $\mathrm{C}^{2} \Sigma_{u}^{+}$potential curve. The second pathway, labelled XUV+IR (II), describes a direct two-photon transition to the dissociative $\mathrm{D}^{2} \Pi_{g}$ state (which has a small cross-section for one-photon XUV photoionization). The main contributing virtual intermediate state for this transition is the $\mathrm{B}^{2} \Sigma_{u}^{+}$state, which is prominent in $\mathrm{N}_{2}$ photoionization and is strongly dipole-coupled to the $\mathrm{D}^{2} \Pi_{g}$ state. Unlike the sequential pathway (I), the direct pathway (II) requires the XUV and IR pulses to overlap. Sequential excitation of the $\mathrm{D}^{2} \Pi_{g}$ state (via the $\mathrm{B}^{2} \Sigma_{u}^{+}$state) requires at least 3 IR photons and was therefore found to be negligible at the IR intensity used in our simulations. A small direct XUV-IR contribution from the $\mathrm{C}^{2} \Sigma_{u}^{+}$to the $\mathrm{D}^{2} \Pi_{g}$ state was not included for simplicity because it does not alter the final result significantly (see Appendix D).

For the pathways I and II to interfere, the photoelectron wavepackets accompanying each path must overlap both in terms of energy and angular momentum. The energy spacing between the $\mathrm{C}^{2} \Sigma_{u}^{+}$and the $\mathrm{D}^{2} \Pi_{g}$ states and the IR frequency are both much smaller than the bandwidth of the attosecond XUV pulse (Fig. 1), thus ensuring a substantial overlap of the photoelectron wavepackets correlated to the two ionization channels. The ungerade $\mathrm{C}^{2} \Sigma_{u}^{+}$ state is accessed by a single photon transition while the gerade $\mathrm{D}^{2} \Pi_{g}$ state is accessed by a two-photon transition. Hence, the photoelectron wavepackets associated with both pathways will have a gerade symmetry and thus, a substantial energy and angular momentum 
overlap. They will however have different photoionization (half-scattering) phases. The actual amount of coherence expected in the experiment (evaluated using TDSE and R-matrix calculations of the photoionization dynamics, see Appendices B and C) was found to be $\sim 0.5$ (where 1 would correspond to perfect coherence).

The overlap of photoelectron wavepackets $\phi(t)$ originating from the photoionization channels I and II will allow the vibronic wavepackets $\Psi(t)$ and thus the corresponding vibrational wavepackets $\chi(t)$, originating from paths I and II, to interfere on the detector. The final KER spectra will depend both on the phase of the photoelectron wavepacket $\phi(t)$, accumulated during the ionization, and the phase of the vibrational wavepacket $\chi(t)$, accumulated during propagation along the paths I and II towards dissociation.

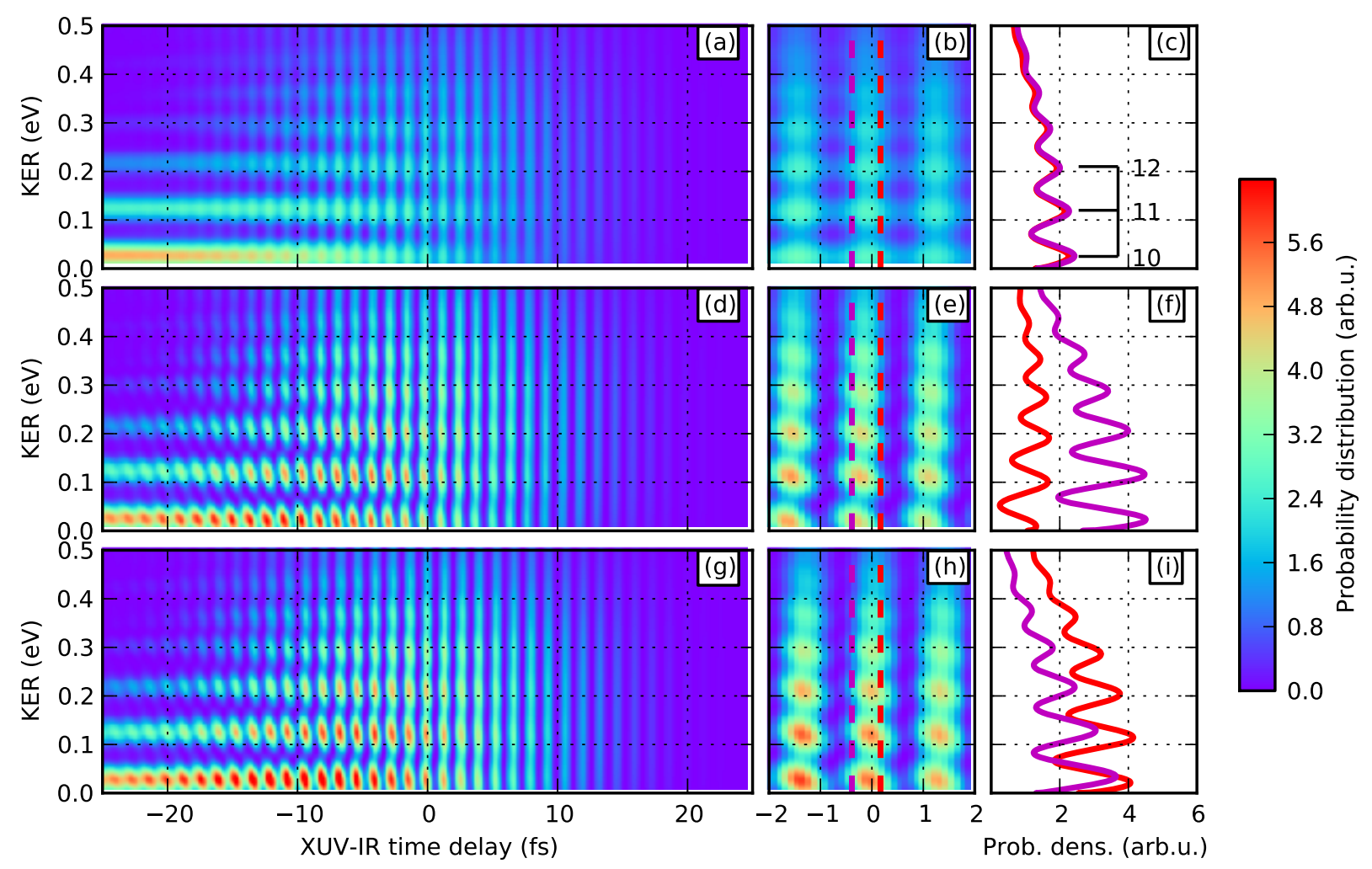

FIG. 2. Calculated $\mathrm{N}^{+}$KER spectra as a function of XUV and IR pump-probe time delay for the case when the summation of direct (path II) and sequential (path I) dissociation channels is incoherent (a) and coherent (d) and (g) with the relative scattering phase set to $\pi / 2$ and 0 , respectively (see text). A zoom of the spectra is shown in (b), (e) and (h). Vertical lines show two selected time delays at which the spectra are plotted in (c), (f) and (i). Negative time delay corresponds to the XUV pulse arriving before the IR pulse. 
To investigate the role of the initial electron coherence on the KER spectra, we solve the Time-Dependent Schrödinger Equation (TDSE) for the vibrational wavepackets $\chi(R, t)$ in the $\mathrm{N}_{2}^{+}$ion in the IR field. We assume two limiting cases for the photoelectron dynamics: no coherence and perfect coherence between the ionization channels. In Eq. 2 this corresponds to $\left\langle\phi_{j}(t) \mid \phi_{i}(t)\right\rangle=0$ and $\mathrm{e}^{-\imath \delta}$ respectively. For the purpose of illustration we chose values 0 and $\pi / 2$ for the scattering phase difference $\delta$ (see Appendices $\mathrm{B}$ and $\mathrm{C}$ for the discussion of actual values of $\delta$ obtained from ab-initio calculations and how they can be manipulated experimentally).

In our simulation, ionic vibrational wavepackets were created as a Franck-Condon (FC) projection of the $\mathrm{N}_{2}$ vibrational ground state at different times during the IR pulse corresponding to the arrival time of the attosecond XUV pulse. One-photon ionization to the $\mathrm{C}^{2} \Sigma_{u}^{+}$state (pathway I) was simulated by placing a vibrational FC wavepacket in the corresponding state. The direct two-photon XUV+IR ionization (pathway II) was simulated by preparing a vibrational FC wavepacket in the IR-dressed $\mathrm{B}^{2} \Sigma_{u}^{+}$state. The coupling between the $\mathrm{B}^{2} \Sigma_{u}^{+}$and $\mathrm{D}^{2} \Pi_{g}$ states by the IR field ensured inclusion of the direct transition between the two states, as well as the effects of the envelope and the XUV-IR delay-dependent phase of the IR field.

The vibrational wavepackets were propagated on the $\mathrm{B}^{2} \Sigma_{u}^{+}, \mathrm{C}^{2} \Sigma_{u}^{+}$and $\mathrm{D}^{2} \Pi_{g} \mathrm{PEC}$ of $\mathrm{N}_{2}^{+}$, coupled by the IR laser pulse with parameters listed previously. The XUV photoionization cross-sections were obtained from literature [19]. The PEC and the transition dipoles were calculated with the GAMESS-US package [21], using an aug-cc-pVTZ basis set. The MRCI (multireference configuration-interaction) wavefunctions included all single excitations from CAS $(9,8)$ (complete active space), which was optimized for the $\mathrm{D}^{2} \Pi_{g}$ state. In addition to the states shown in Figure 1, many more electronic states are present in the cation. However, due to the ionization dipole selection rules, their energetic positions and their weaker couplings to the $\mathrm{C}^{2} \Sigma_{u}^{+}$and $\mathrm{B}^{2} \Sigma_{u}^{+}$states, they were neglected. Spectroscopically, the $\mathrm{D}^{2} \Pi_{g}$ state of $\mathrm{N}_{2}^{+}$is known to arise from a strongly-interacting pair of ${ }^{2} \Pi_{g}$ states [19]. For simplicity, we chose to include only the lower adiabatic branch of this pair in our simulations. It is responsible for the de-excitation step of the sequential path, and is therefore sufficient to reproduce qualitative features of the dissociation paths I and II. The vibrational wavepacket propagation on the IR-field-coupled $\mathrm{B}^{2} \Sigma_{u}^{+}, \mathrm{C}^{2} \Sigma_{u}^{+}$and $\mathrm{D}^{2} \Pi_{g}$ PEC was performed using a standard split-operator method. The final $\mathrm{N}^{+}$KER spectrum was extracted by projection 
onto the continuum eigenstates of the $\mathrm{D}^{2} \Pi_{g}$ PEC.

Calculated KER spectra as a function of the XUV-IR pump-probe delay are shown in Fig. 2. In the (a-c) panels, the contributions from the XUV-IR (I) and XUV+IR (II) dissociation pathways are added up incoherently. In the (d-f) panels they are added coherently, with the difference of the photoelectron scattering phases between different ionization channels set to $\delta=\pi / 2$ whereas in the (g-i) panels, the phase is set to $\delta=0$. The KER spectra show peaks corresponding to de-excitation of vibrational states of the $\mathrm{C}^{2} \Sigma_{u}^{+}$state, with $v>10$, (pathway I). The $v=10$ vibrational state is above the $L_{1}$ dissociation limit $\left(E_{L_{1}}=24.293 \mathrm{eV}\right)$ by an energy which is slightly bigger than the energy of a single IR photon. Therefore, this vibrational state is the first to show up in the KER spectra. Note that small computational inaccuracies in the vibrational progression of the $\mathrm{C}^{2} \Sigma_{u}^{+}$state are amplified in the KER spectra because the high lying vibrational states have a KER which is close to zero. An additional shift appears because the transition matrix elements for the vibrational states strongly depend on energy. Lower vibrational levels of the $\mathrm{C}^{2} \Sigma_{u}^{+}$state do not contribute, since they are de-excited onto the bound part of the $\mathrm{D}^{2} \Pi_{g}$ PEC. The width of the vibrational lines reflects the bandwidth of the IR pulse, since IR-induced de-excitation is the only decay mechanism present in the calculation.

Clear differences can be seen between the KER spectra for incoherent and coherent addition. Although in both cases a modulation of the spectra is observed at twice the IR frequency, its origin is rather different. In the case of incoherent addition, the modulation of the spectra is entirely due to the sub-cycle dependence of direct pathway II, which follows the oscillating IR electric field at the moment of ionization [12]. The modulation depth is highest near the peak of the IR pulse. The vibrational band originating from sequential pathway I is not modulated, and is simply superimposed on the oscillations caused by pathway II. The contribution from pathway I becomes stronger as one moves from positive to negative time delays, because the sequential path requires that XUV ionization precedes the IR de-excitation. The maxima of the oscillation coincide with maxima and minima of the IR field (see Fig. 2b).

In the coherent case (Figure 2d) the contrast in the modulation is enhanced, especially near $\Delta t=-10$ fs when the contributions from pathways I and II are similar. Unlike the incoherent case, the maxima of the oscillations do not match the maxima and minima of the IR electric field but are delayed by $\Delta t \sim 200$ as (see Fig. 2e). 
The observed beating in the coherent case is due to an interference between the direct $\mathrm{XUV}+\mathrm{IR}$ (II) and the sequential XUV-IR (I) dissociation pathways. This process is analogous to the common RABBITT scheme, where the relative delay between the attosecond XUV pulse train and the IR pulse controls the constructive and destructive interference of photoelectron sidebands [22], see also [12, 23]. Here the XUV-IR time delay does not modulate the continuum population and/or induce free-free transitions. Instead the delay controls the relative electronic phase between the direct (II) and sequential (I) dissociation pathways, leading to modulation of the vibrationally resolved $\mathrm{N}^{+}$KER. The 200 as time offset of the beating pattern arises as a result of the phase difference between the two paths. It encodes (i) the initial difference between the photoionization scattering phases in the two different continua and (ii) the phase associated with the vibrational motion in the sequential pathway.

Figures $2 \mathrm{c}$ and $2 \mathrm{f}$ show vibrationally resolved $\mathrm{N}^{+}$KER spectra at two selected time delays. In the incoherent case (Fig. 2c) the vibrational peaks resulting from $\mathrm{C}^{2} \Sigma_{u}^{+}$to $\mathrm{D}^{2} \Pi_{g}$ de-excitation occur at the same energy for both time delays. In the coherent case the maxima observed in the KER change with the time delay, with a shift that depends on the scattering phase (Fig. 2f and i). This effect reflects the fact that the change of the time delay also modifies the vibrational dynamics.

Since the vibrational motion in the cation can be reliably calculated, the KER spectra can be used to extract the initial electronic coherence established by photoionization. This is illustrated in the (g-i) panels of Fig. 2, where the electronic photoionization phase was changed by 90 degrees. A clear impact is seen in the KER spectra, changing both the offset of the beating pattern and the appearance energies of the vibrational peaks.

In conclusion, we have shown that quantum entanglement between the photoelectron and the molecular ion, established during photoionization, can influence the outcome of a photochemical reaction that evolves on a timescale of tens of femtoseconds. Using $\mathrm{N}_{2}$ as a model system, we have shown that vibrationally resolved KER spectra depend on the interplay of attosecond electronic dynamics during ionization and femtosecond vibrational dynamics that leads to fragmentation of the molecule. As long as the vibrational dynamics can be reliably simulated, XUV-IR delay-dependent KER spectra can be used to extract the relative photoionization phases between different ionic channels. This would allow to establish the initial shape, location and momentum of the hole created by ionization. 


\section{ACKNOWLEDGMENTS}

We would like to thank Jochen Mikosch for valuable discussions and suggestions. We acknowledge financial support of the Marie Curie ITN networks CORINF and ATTOFEL and of the EPSRC Programme Grant "Attosecond Electron Dynamics in Molecular and Condensed Matter".

\section{Appendix A: Describing the photoelectron wavepacket and the residual ion dynam- ics using the Time-Dependent Schrödinger Equation}

We are describing the dynamics after ionizing the $\mathrm{N}_{2}$ molecule with a two-color XUV+IR fields. The wavefunction to describe the photoelectron and the residual ion after photoionization is expressed in the close-coupling form

$$
\left|\Psi\left(r_{1} \ldots r_{n}, R, t\right)\right\rangle=\sum_{m}\left|\phi_{m}\left(r_{n}, R, t\right)\right\rangle\left|\Psi_{m}\left(r_{1} \ldots r_{n-1}, R, t\right)\right\rangle
$$

where the summation is over all the ionization channels $m .\left|\phi_{m}\left(r_{n}, R, t\right)\right\rangle$ is the photoelectron wavepackets correlated to the vibronic wavefunction $\left|\Psi_{m}\left(r_{1} \ldots r_{n-1}, R, t\right)\right\rangle$ associated with the $m$-th ionization channel. The coordinate $r_{n}$ is associated to the photoelectron, while the coordinates $r_{1}, \ldots, r_{n-1}$ are associated to all the electrons that remain bound in the residual ion. $R$ is the internuclear distance.

The ansatz in Eq. A1 is exact in the asymptotic region, i.e., when the interaction between the photoelectron and the ion can be neglected and is often used to describe the asymptotic region in scattering and photoionization calculations (see e.g. review in [24]). It can be applied for our investigation, i.e., to describe the system after the photoionization is over.

Note, that the ansatz in Equation A1 neglects the antisymmetrization between the departing photoelectron $r_{n}$ and the remaining bound electrons $r_{1}, \ldots, r_{n-1}$ and is also over

complete for an n-electron system. These features would become important in describing the photoionization process, when the photoelectron is still close to the ionic core.

Using the ansatz in Equation A1 the time-dependent Schödinger equation (TDSE) can 
be written as

$$
\begin{aligned}
& \imath\left(\frac{\partial}{\partial t}\left|\phi_{m}\right\rangle\right)\left|\Psi_{m}\right\rangle+\imath\left|\phi_{m}\right\rangle\left(\frac{\partial}{\partial t}\left|\Psi_{m}\right\rangle\right)= \\
& \sum_{m^{\prime}}\left(\hat{H}_{\chi}\left|\phi_{m^{\prime}}\right\rangle\right)\left|\Psi_{m^{\prime}}\right\rangle+\sum_{m^{\prime}}\left(\left[\hat{H}_{i}+\hat{V}_{i r}\right]\left|\Psi_{m^{\prime}}\right\rangle\right)\left|\phi_{m^{\prime}}\right\rangle+\hat{V}_{x u v}\left|\Psi_{0}\right\rangle
\end{aligned}
$$

where $\hat{H}_{\chi}\left(r_{1}, \ldots, r_{n}, R, t\right)$ is the Hamiltonian governing the photoelectron and containing the interaction between the photoelectron $\left|\phi_{m}\left(r_{n}, R, t\right)\right\rangle$ and the residual ion $\left|\Psi_{m}\left(r_{1} \ldots r_{n-1}, R, t\right)\right\rangle$. $\hat{H}_{i}\left(r_{1}, \ldots, r_{n-1}, R, t\right)$ is the Hamiltonian governing the dynamics in the residual ion and $\left|\Psi_{0}\left(r_{1}, \ldots, r_{n}, R, t\right)\right\rangle$ is the wavefunction of the initial state, which in our case is the $\mathrm{N}_{2}$ ground state. We further assumed that the XUV pulse, with corresponding potential operator $\hat{V}_{\text {xuv }}(t)$ is only responsible for ionization, while the IR pulse, with corresponding potential operator $\hat{V}_{i r}(t)$, interacts only with the ionic core. Hence, we do not include the dressing of the photoionization continuum by the IR field. This latter process leads to the well-studied RABBITT type interference in the continuum, but does not change the final state of the cation, which we are interested in here. However, the TDSE in Equation A2 includes direct two-photon XUV+IR ionization, when the XUV photon is absorbed by the photoelectron and the IR photon is absorbed by the ion core.

Following the work in [25] Equation A2 is re-written as two coupled equations:

$$
\begin{aligned}
\imath\left(\frac{\partial}{\partial t}\left|\phi_{m}\right\rangle\right)\left|\Psi_{m}\right\rangle & =\sum_{m^{\prime}}\left(\hat{H}_{\chi}\left|\phi_{m^{\prime}}\right\rangle\right)\left|\Psi_{m^{\prime}}\right\rangle+\hat{V}_{x u v}\left|\Psi_{0}\right\rangle \\
\imath\left|\phi_{m}\right\rangle\left(\frac{\partial}{\partial t}\left|\Psi_{m}\right\rangle\right) & =\sum_{m^{\prime}}\left|\phi_{m^{\prime}}\right\rangle\left(\left[\hat{H}_{i}+\hat{V}_{i r}\right]\left|\Psi_{m^{\prime}}\right\rangle\right) .
\end{aligned}
$$

Assuming that the timescale of photoionization by an attosecond XUV pulse is much faster than the timescale of the nuclear dynamics in the residual ion, Equation A3 can be solved for a fixed internuclear distance, neglecting the vibrational dynamics. This allows one to solve Equation A4 separately where the photoelectron wavepackets $\left|\phi_{m}\right\rangle$ become "time-dependent coefficients" for dynamics in the ion. The non-adiabatic effects, i.e., the effects of the nuclear kinetic energy operator on the photoelectron wavepackets $\left|\phi_{m}\right\rangle$ during photoionization, are neglected. We further assume that photoionization is fast and that the photoelectron travels far away from the ion so that the interaction between the two is negligible once the vibrational dynamics in the ion starts. In this case $\left|\phi_{m}\right\rangle$ in Equation A4 become time-independent initial coefficients for the dynamics in the ion. 
In this work we do not solve the photoionization dynamics described by Equation A3. Instead we estimate the photoelectron wavepackets, integrated over all final photoelectron energies, as

$$
\int \mathrm{d} E\left\langle\phi^{E} \mid \phi_{m}\right\rangle=\sqrt{\sigma_{m}} \cdot \mathrm{e}^{-\imath \gamma}
$$

where $\sigma_{m}$ is the photoionization cross-section extracted from experimental measurements found in the literature (see the main text of the manuscript), and $\gamma$ is the phase.

This work investigates the interference of two ionization channels. To illustrate the effect of the photoionization phase $\gamma$ in the dissociation process, two values of phase difference $\delta=\gamma_{C}-\gamma_{D}=0$ and $\pi / 2$ are considered. Furthermore, an evaluation of the phase difference between the ionization channels that are studied in this work is presented in Sections II and III below.

Finally, we insert Equation A5 into Equation A4 as initial conditions for the ionic dynamics, which is justified when the photoionization is much faster than vibrational dynamics in the ion. Finally, we solve Equation A4 numerically with the described initial condition. Hence, the calculation fully includes the IR field, but does not explicitly include the XUV field.

\section{Appendix B: Estimate for photoelectron wavepacket overlap using single-photon} ionization dipoles

Let us consider two photoionization channels: channel I represents single photon ionization into the $\mathrm{C}^{2} \Sigma_{u}^{+}$state and channel II represents two photon XUV+IR ionization into the $\mathrm{D}^{2} \Pi_{g}$ state of $\mathrm{N}_{2}^{+}$molecule. In addition, let us consider the case when both XUV and IR pulses are oriented perpendicular to the molecular axis.

Let us further assume that in channel II the XUV photon is absorbed by the photoelectron while the IR photon is absorbed by the residual ion. Hence, we will assume that the photoelectron wavepacket can be represented using the dipoles for photoionization into the $\mathrm{B}^{2} \Sigma_{u}^{+}$state while the IR photon changes the final target state into the $\mathrm{D}^{2} \Pi_{g}$ state. Using the close-coupling ansatz introduced in the Equation A1 the wavefunction after photoionization is given by

$$
|\Psi\rangle=\left|\phi_{D}\right\rangle\left|\Psi_{D}\right\rangle+\left|\phi_{C}\right\rangle\left|\Psi_{C}\right\rangle
$$


where $\left|\Psi_{C}\right\rangle$ and $\left|\Psi_{D}\right\rangle$ are the vibronic states of the $\mathrm{N}_{2}^{+}$ion and $\left|\phi_{C, D}\right\rangle$ are the corresponding photoelectron wavepackets. We can define the density matrix of the system as

$$
\rho=\left\langle\epsilon\left|\left\langle\Psi_{C, D} \mid \Psi\right\rangle\left\langle\Psi \mid \Psi_{C, D}\right\rangle\right| \epsilon\right\rangle
$$

where $|\epsilon\rangle$ is a photoelectron (single electron) eigenstate with an asymptotic energy $\epsilon$, and where $\left|\Psi_{C}\right\rangle$ and $\left|\Psi_{D}\right\rangle$ are assumed to be orthonormal.

The amount of coherence $g$ between the $\left|\Psi_{C}\right\rangle$ and $\left|\Psi_{D}\right\rangle$ ionization channels can be quantified by the off-diagonal elements of the reduced density matrix $\rho$

$$
g=\frac{\rho_{C D}}{\sqrt{\rho_{A} \cdot \rho_{D}}}=\int \mathrm{d} \epsilon \frac{\left\langle\epsilon \mid \phi_{D}\right\rangle\left\langle\phi_{C} \mid \epsilon\right\rangle}{\sqrt{\left|\phi_{D}\right|^{2} \cdot\left|\phi_{C}\right|^{2}}}=\frac{\left\langle\phi_{C} \mid \phi_{D}\right\rangle}{\sqrt{\left|\phi_{D}\right|^{2} \cdot\left|\phi_{C}\right|^{2}}}
$$

and is directly related to the overlap of the photoelectron wavepackets correlated to each channel $\left\langle\phi_{C} \mid \phi_{D}\right\rangle$.

\begin{tabular}{c|c||c|c}
\hline \multicolumn{2}{c||}{ perpendicular } & \multicolumn{2}{c}{ parallel } \\
\hline channel & coherence & channel & coherence \\
$(\mathrm{l}, \mathrm{m})$ & $\left|g_{l, m}\right|$ & $(\mathrm{l}, \mathrm{m})$ & $\left|g_{l, m}\right|$ \\
\hline$(2,1)$ & $0.77(0.97)$ & $(2,0)$ & $0.89(0.93)$ \\
$(4,1)$ & $0.46(0.68)$ & $(0,0)$ & $0.45(0.73)$ \\
$(4,3)$ & $0.51(0.74)$ & $(4,0)$ & $0.76(0.81)$ \\
\hline
\end{tabular}

TABLE I. Amount of coherence between considered ionization channels for each partial wave for an XUV field oriented perpendicular and parallel to the molecular axis. The channels are arranged in an order of decreasing strength. The values in brackets are for dipoles with removed continuum resonances.

We have used the R-matrix method [26] to obtain the single-photon ionization dipoles into the $\mathrm{C}^{2} \Sigma_{u}^{+}$and $\mathrm{B}^{2} \Sigma_{u}^{+}$(intermediate) states resolved in final energy and (l, m)-partial wave channel (see Figure B.1):

$$
d^{C, B}(\epsilon, l, m)=\sum_{l, m} a_{l, m}^{C, B}(\epsilon) Y_{l, m}
$$

where $Y_{l, m}$ are spherical harmonic functions (see ref. [26] for more details). The dipoles are calculated at the equilibrium internuclear distance. 

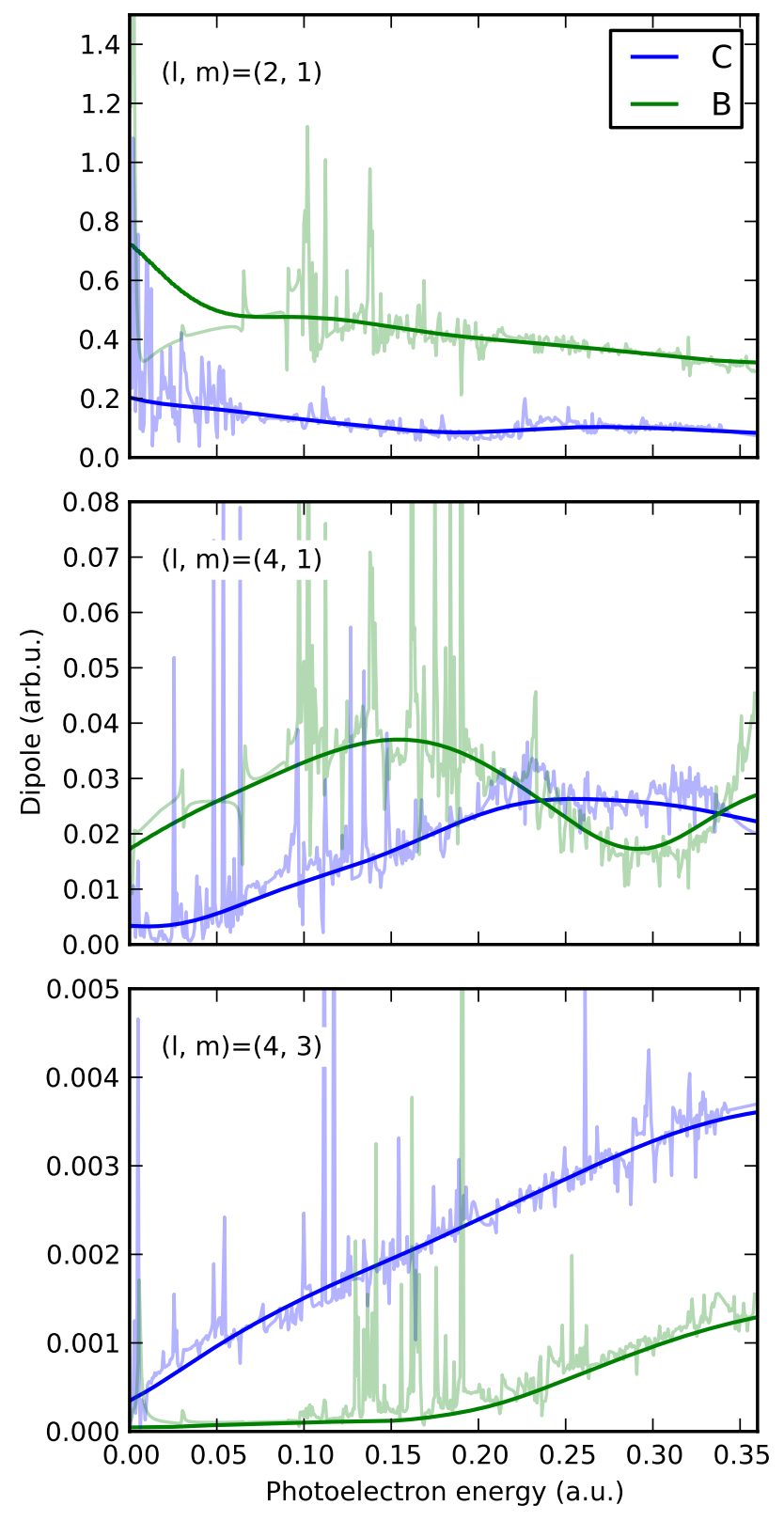

FIG. B.1. Single photon ionization dipoles into the $\mathrm{C}^{2} \Sigma_{u}^{+}$(blue) and $\mathrm{B}^{2} \Sigma_{u}^{+}$(green) target states of the $\mathrm{N}_{2}^{+}$ion and for different final $(1, \mathrm{~m})$ partial waves for laser polarization perpendicular to the molecular axis. Sharp peaks present in the dipoles, which are due to the continuum resonances, are removed by a Gaussian filter of width 0.07 a.u. (bold lines).

In the R-matrix calculation, the dipoles are calculated between the $\mathrm{N}_{2}$ ground state and 
outgoing scattering states $\left|\phi^{-}\right\rangle$. In this case Equation B3 becomes

$$
g=\frac{\rho_{C D}}{\sqrt{\rho_{A} \cdot \rho_{D}}}=\iint \mathrm{d} \epsilon_{C} \mathrm{~d} \epsilon_{D} \frac{\left\langle\phi_{C}^{-} \mid \epsilon_{C}\right\rangle\left\langle\epsilon_{C} \mid \epsilon_{D}\right\rangle\left\langle\epsilon_{D} \mid \phi_{D}^{-}\right\rangle}{\sqrt{\left|\phi_{D}^{-}\right|^{2} \cdot\left|\phi_{C}^{-}\right|^{2}}} \approx \int \mathrm{d} \epsilon \frac{\left\langle\phi_{C}^{-} \mid \epsilon\right\rangle\left\langle\epsilon \mid \phi_{D}^{-}\right\rangle}{\sqrt{\left|\phi_{D}^{-}\right|^{2} \cdot\left|\phi_{C}^{-}\right|^{2}}}
$$

where we have assumed that $\left\langle\epsilon_{C} \mid \epsilon_{D}\right\rangle=\delta\left(\epsilon_{C}-\epsilon_{D}\right)$, which is necessary to use the photoionization dipoles obtained from R-matrix calculation to estimate the coherence between the two channels. This approximation is more accurate for high photoelectron energy values $\epsilon$. For low values of $\epsilon$, the amount of coherence, estimated below, will provide an upper bound. Note also, that the approximation above does not influence the accuracy of the dipoles, but only the estimate of coherence between the photoionization channels.

Using the dipoles in Equation B4, Equation B5 can be written for each final $(1, \mathrm{~m})$ partial wave as

$$
g_{l, m}=\sum_{\epsilon}\left(a_{l, m, \epsilon}^{B}\right)^{*} a_{l, m, \epsilon}^{C} / \sqrt{\sum_{\epsilon}\left|a_{l, m, \epsilon}^{B}\right|^{2} \cdot \sum_{\epsilon}\left|a_{l, m, \epsilon}^{C}\right|^{2}} .
$$

Note that, as mentioned earlier, the dipoles for single photon ionization into the $\mathrm{B}^{2} \Sigma_{u}^{+}$state are used to quantify the two-photon ionization into the $\mathrm{D}^{2} \Pi_{g}$ state.

For single photon ionization into the $\mathrm{B}^{2} \Sigma_{u}^{+}$and $\mathrm{C}^{2} \Sigma_{u}^{+}$final target states, and for an XUV field oriented perpendicular to the molecular axis, only $3(1, \mathrm{~m})$ channels contribute significantly. The amount of coherence described by Equation B6 for these channels is summarized in table I. The estimate suggest that the amount of coherence created between the photoionization channels I and II can be as large as 0.75 .

The high level of coherence between the two photoionization channels can be well understood by inspecting their phase difference as a function of energy, expressed by the function

$$
\cos \left(\delta_{l, m}(\epsilon)\right)=\frac{\left(a_{l, m, \epsilon}^{B}\right)^{*} a_{l, m, \epsilon}^{C}+\text { c.c. }}{2\left|a_{l, m, \epsilon}^{B}\right|\left|a_{l, m, \epsilon}^{C}\right|}
$$

which is plotted in Fig. B.2. Despite sharp jumps in the phase, the average phase difference over a small energy region is approximately constant and changes only slowly with photoelectron energy. This means that, when contributions to all the photoelectron energies are summed up, the phase variation does not wash out the interferences.

In an experiment the photon energy and bandwidth of the laser pulse will define the photoelectron energy "window" for each of the channels. From Fig. B.2 it can be seen that it is possible to control the photoionization phase difference by choosing such a "window" around different photoelectron energies. 


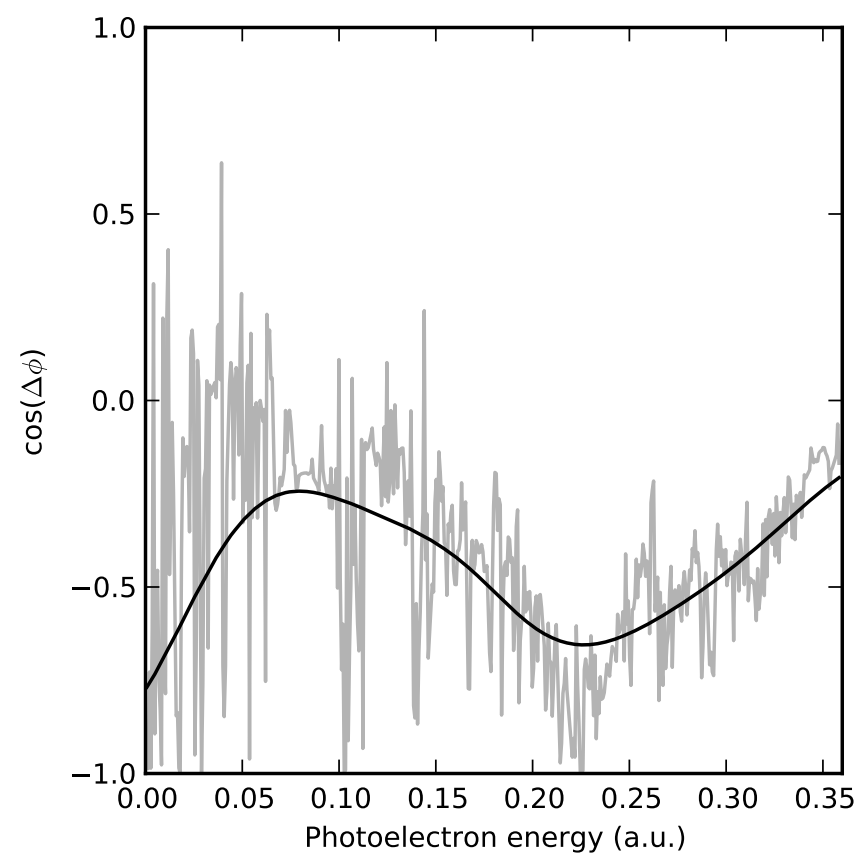

FIG. B.2. Cosine of the phase difference between the dipoles for photoionization into the $\mathrm{C}^{2} \Sigma_{u}^{+}$ and $\mathrm{B}^{2} \Sigma_{u}^{+}$states as a function of photoelectron energy. The phase is shown for the dominant $(1, m)=(2,1)$ partial wave and for the XUV field perpendicular to the molecular axis. The phase difference for dipoles that include the continuum resonances (gray line) and without the continuum resonances (black line) are shown.

\section{Appendix C: Estimate of photoelectron wavepacket overlap from a time-dependent} photoionization calculation

We estimate the overlap of the photoelectron wavepackets for ionization of $\mathrm{N}_{2}$ with a short XUV pulse, by solving the time-dependent Schrödinger equation (TDSE) for the electronic dynamics following the procedure in [27]. In this approach, a set of coupled TDSE are solved to describe the neutral amplitude and the continuum electron correlated to different cationic states, coupled to each other by electron-electron interaction and the laser field. It uses a field-free multielectron wave function ansatz for the cationic and neutral states (similarly to the ansatz in Equation A1) while the photoelectron wavepacket is calculated on a spatial Cartesian grid. The coherence between different ionization channels is estimated by calculating the overlap between the photoelectron wavepackets associated to the final $\mathrm{C}$ 
$\left(2^{2} \Sigma_{u}^{+}\right)$and $\mathrm{D}\left(1^{2} \Pi_{g}\right)$ cation states:

$$
g=\frac{\left\langle\phi_{D} \mid \phi_{C}\right\rangle}{\left(\left\langle\phi_{D} \mid \phi_{D}\right\rangle\left\langle\phi_{C} \mid \phi_{C}\right\rangle\right)^{1 / 2}}
$$

at the end of the XUV pulse.

We are using $\mathrm{N}_{2}$ at the equilibrium neutral geometry $(\mathrm{R}=1.09768 \AA)$. For the electronic structure calculations we use the aug-cc-pVTZ basis set and large-core Stuttgart ECPs on nitrogen. Wavefunctions are obtained using MR-CIS, with the minimal valence CAS (9e, 8o) optimized for the lowest ${ }^{2} \Pi_{g}$ state of the cation. Neutral and cationic wavefunctions are constructed from the same single-particle orbitals optimized for the cation. Calculated vertical IPs were adjusted by $+1.217 \mathrm{eV}$ to match the experimental value of first IP.

The effects of the IR were simulated by adding a static field of strength $F=0.0169$, which corresponds to an IR peak intensity of $10^{13} \mathrm{~W} / \mathrm{cm}^{2}$. A Gaussian XUV pulse with 500 as duration (FWHM) and $10^{12} \mathrm{~W} / \mathrm{cm}^{2}$ intensity was used in the simulation. Both XUV and IR field were polarized in the same direction, perpendicularly to the molecular axis. Note, that the high XUV intensity was used to improve the accuracy of the calculation by enhancing the photoionization signal. For the XUV intensity used, multiphoton effects were still negligible.

The simulation was performed in a 3D box with 82.8 Bohr sides, using a Cartesian product grid with 0.18 Bohr resolution and 0.003 au time step in the leap-frog propagator. The XUV pulse was centred at 40 au time (about $1 \mathrm{fs}$ ). Several calculations were performed using harmonics of a $795 \mathrm{~nm}$ IR light $(1.56 \mathrm{eV})$ between $\mathrm{H} 14$ and $\mathrm{H} 18$ as the central frequency of the XUV field.

Snapshots of the continuum part of the wavefunction (with the Dyson orbital projected out; see [27] for the exact separation procedure) in the $\mathrm{D}\left(1^{2} \Pi_{g}\right)$ and $\mathrm{C}\left(2^{2} \Sigma_{u}^{+}\right)$final ionization channels are shown in Figure C.1. The snapshots were taken at 80 au time. In all cases, absorption of the continuum state at the grid edges was negligible.

The degree of coherence and the relative phase of the continuum were both calculated using the entire grid, and with the central region (soft-sphere, about 16 Bohr in diameter) masked out. The results are presented in table II. 


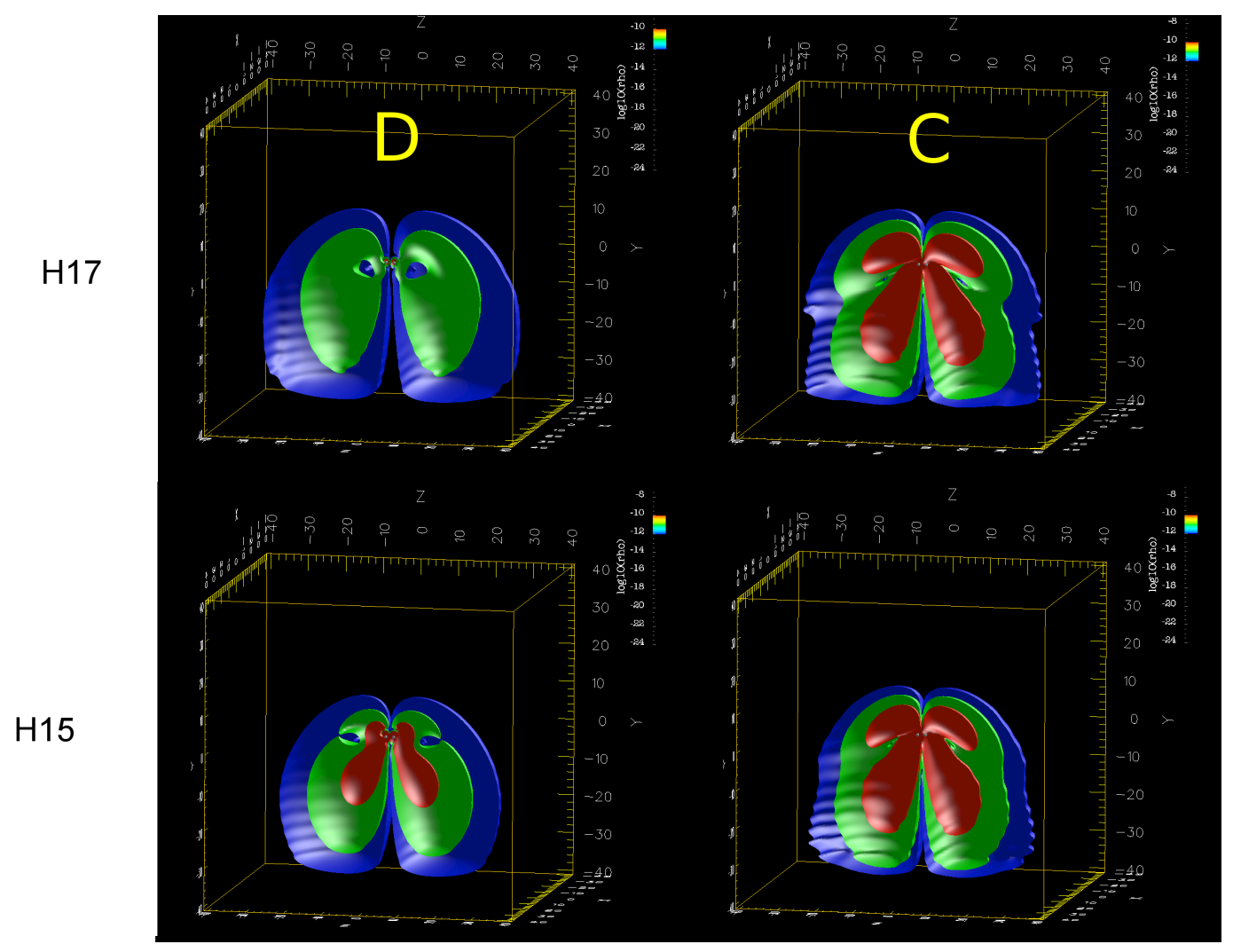

FIG. C.1. Snapshots of continuum wavepackets in the D $\left(1^{2} \Pi_{g}\right)$ and $\mathrm{C}\left(2^{2} \Sigma_{u}^{+}\right)$ionization channels for a 500 as XUV pulse with energy centred around harmonic H15 and H17 of the IR field.

\begin{tabular}{c|cc|cc}
\multirow{2}{*}{ harmonic } & \multicolumn{2}{|c|}{ overlap } & \multicolumn{2}{c}{ masked overlap } \\
& $|g|$ & $\operatorname{Arg}[\mathrm{g}]$ & $|g|$ & $\operatorname{Arg}[\mathrm{g}]$ \\
\hline 14 & 0.16 & $175^{\circ}$ & 0.60 & $141^{\circ}$ \\
15 & 0.28 & $140^{\circ}$ & 0.55 & $140^{\circ}$ \\
16 & 0.34 & $135^{\circ}$ & 0.48 & $140^{\circ}$ \\
17 & 0.32 & $133^{\circ}$ & 0.38 & $138^{\circ}$ \\
18 & 0.26 & $130^{\circ}$ & 0.29 & $133^{\circ}$ \\
\hline
\end{tabular}

TABLE II. The degree of coherence $|g|$ between photoelectron wavepackets originating from the C $\left(2^{2} \Sigma_{u}^{+}\right)$and the $\mathrm{D}\left(1^{2} \Pi_{g}\right)$ photoionization channels for a 500 as XUV pulses with carrier frequency centred around harmonic 14 to harmonic 18 of a $795 \mathrm{~nm}$ driving IR pulse. Additionally, a static electric field is present in the simulation to account for the effects of the dressing IR field. 


\section{Appendix D: Separation of direct and sequential transitions}

The Franck-Condon (FC) region of the $\mathrm{N}_{2}$ molecule is located at an internuclear distance at which the $\mathrm{C}^{2} \Sigma_{u}^{+}$and $\mathrm{D}^{2} \Pi_{g}$ potentials of $\mathrm{N}_{2}^{+}$are energetically very close to each other. However, this is not an avoided crossing, as the two states have different symmetries. The coupling of the $\mathrm{C}^{2} \Sigma_{u}^{+}$and $\mathrm{D}^{2} \Pi_{g}$ states by the IR field near the FC point induces noticeable two-photon XUV-IR direct transition to the $\mathrm{D}^{2} \Pi_{g}$ state, where the $\mathrm{C}^{2} \Sigma_{u}^{+}$state acts as an intermediate virtual state. The strength of this transition strongly depends on the exact energy difference between the $\mathrm{C}^{2} \Sigma_{u}^{+}$and $\mathrm{D}^{2} \Pi_{g}$ potential curves. This energy difference is in turn sensitive to the accuracy of the calculated PEC.

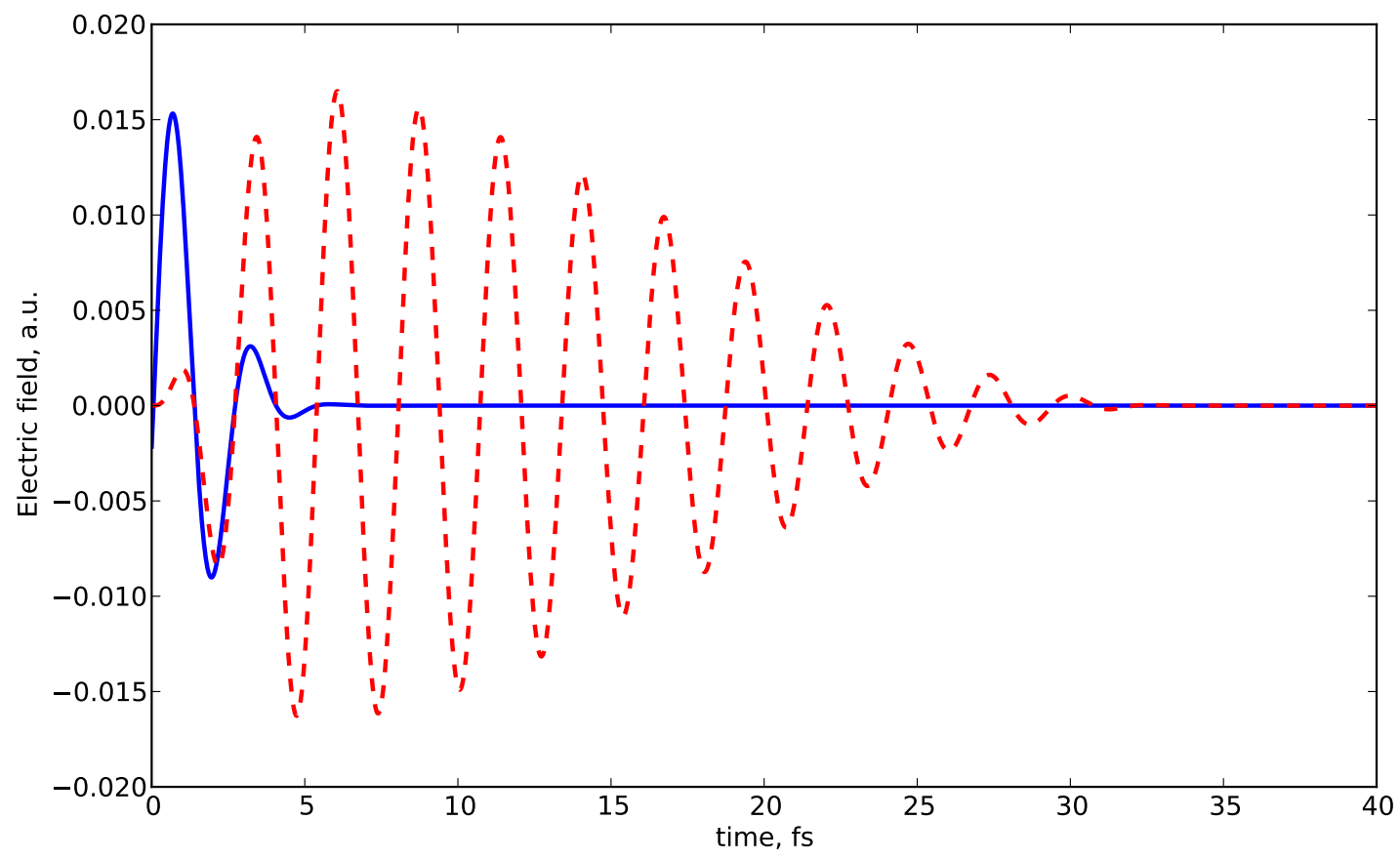

FIG. D.1. Laser pulse splitting to separate the direct (blue full line) from the sequential (red dashed line) transitions; $\mathrm{t}=0$ corresponds to the chosen arrival time of the XUV pulse.

We have calculated the contribution from the direct transition from the $\mathrm{C}^{2} \Sigma_{u}^{+}$to the $\mathrm{D}^{2} \Pi_{g}$ state and evaluated its influence on the final Kinetic Energy Release (KER) spectra as a function of time-delay between the XUV and IR pulses. In order to separate the direct from the sequential transitions, to the $\mathrm{D}^{2} \Pi_{g}$ state, the IR laser pulse was split into two parts: (i) leading edge, that induces only direct transitions and (ii) trailing edge, that induces only 
sequential transitions (Figure D.1). The KER from the $\mathrm{D}^{2} \Pi_{g}$ state was then calculated using (i) only the direct $\mathrm{B}^{2} \Sigma_{u}^{+}$to $\mathrm{D}^{2} \Pi_{g}$ and sequential $\mathrm{C}^{2} \Sigma_{u}^{+}$to $\mathrm{D}^{2} \Pi_{g}$ channels (see Figure 2 in the main text), and (ii) with the direct $\mathrm{C}^{2} \Sigma_{u}^{+}$to $\mathrm{D}^{2} \Pi_{g}$ channel included (Figure D.2).

The KER spectra are only weakly affected by the direct $\mathrm{C}^{2} \Sigma_{u}^{+}$to $\mathrm{D}^{2} \Pi_{g}$ transition. It mainly alters the modulation depth of the time-delay dependent KER spectra and induces a small but observable phase shift to the oscillations of the KER. Note, that the time delay at which the panels (c, f and d) in Fig. D.2 are plotted were also adjusted to this phase shift. Moreover, quantitative corrections to the phase of the oscillation of the KER spectra remain small, because the direct $\mathrm{C}^{2} \Sigma_{u}^{+}$to $\mathrm{D}^{2} \Pi_{g}$ transition is weaker than the other dissociation channels.

Any additional dissociation channels that interfere with the main channels I and II, e.g., the channel described above, will contribute to the phase of the oscillations of the KER spectra in a time-delay measurement. Hence, the $\mathrm{N}^{+}$fragment KER spectra and vibrational peak positions will be sensitive to the scattering phase differences between all the participating channels.
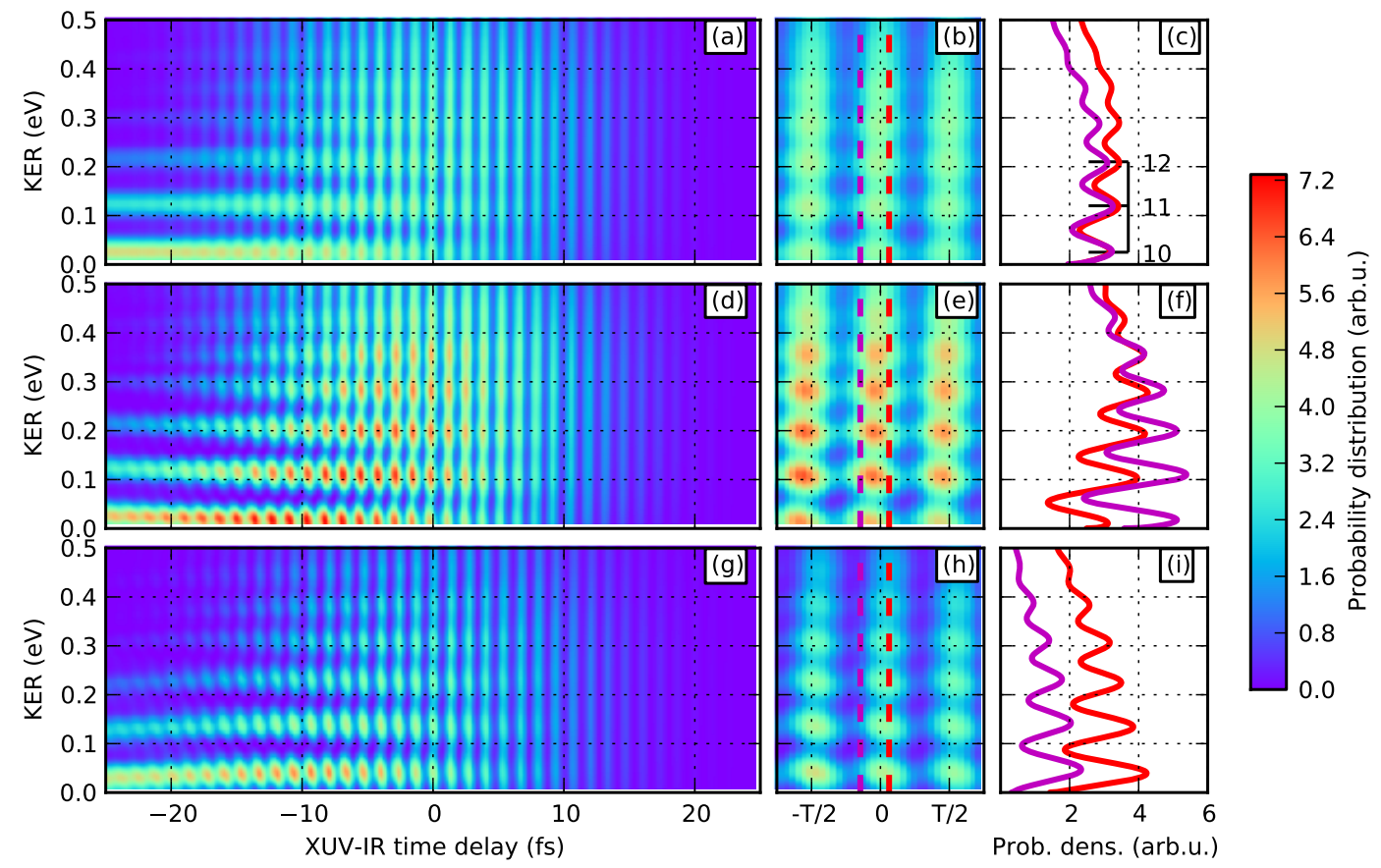

FIG. D.2. $\mathrm{N}^{+}$KER spectra for direct $\mathrm{B}^{2} \Sigma_{u}^{+}$to $\mathrm{D}^{2} \Pi_{g}$, direct $\mathrm{C}^{2} \Sigma_{u}^{+}$to $\mathrm{D}^{2} \Pi_{g}$ and sequential $\mathrm{C}^{2} \Sigma_{u}^{+}$ to $\mathrm{D}^{2} \Pi_{g}$ transitions; see main text for the explanation of the panels. 
[1] F. Krausz and M. Ivanov, Reviews of Modern Physics 81, 163 (2009).

[2] A. I. Kuleff, J. Breidbach, and L. S. Cederbaum, The Journal of Chemical Physics 123, $044111(2005)$.

[3] A. I. Kuleff and L. S. Cederbaum, Chemical Physics 338, 320 (2007).

[4] F. Remacle and R. D. Levine, Proceedings of the National Academy of Sciences of the United States of America 103, 6793 (2006).

[5] R. Weinkauf, E. W. Schlag, T. J. Martinez, and R. D. Levine, The Journal of Physical Chemistry A 101, 7702 (1997).

[6] F. Remacle and R. D. Levine, The Journal of Chemical Physics 110, 5089 (1999).

[7] M. F. Kling, C. Siedschlag, A. J. Verhoef, J. I. Khan, M. Schultze, T. Uphues, Y. Ni, M. Uiberacker, M. Drescher, F. Krausz, and M. J. J. Vrakking, Science (New York, N.Y.) 312, 246 (2006).

[8] P. Dietrich, M. Y. Ivanov, F. A. Ilkov, and P. B. Corkum, Physical Review Letters 77, 4150 (1996).

[9] M. Thachuk, M. Y. Ivanov, and D. M. Wardlaw, The Journal of Chemical Physics 105, 4094 (1996).

[10] M. Thachuk, M. Y. Ivanov, and D. M. Wardlaw, The Journal of Chemical Physics 109, 5747 (1998).

[11] G. Sansone, F. Kelkensberg, J. F. Pérez-Torres, F. Morales, M. F. Kling, W. Siu, O. Ghafur, P. Johnsson, M. Swoboda, E. Benedetti, F. Ferrari, F. Lépine, J. L. Sanz-Vicario, S. Zherebtsov, I. Znakovskaya, A. L'huillier, M. Y. Ivanov, M. Nisoli, F. Martín, and M. J. J. Vrakking, Nature 465, 763 (2010).

[12] F. Kelkensberg, W. Siu, J. F. Pérez-Torres, F. Morales, G. Gademann, A. Rouzée, P. Johnsson, M. Lucchini, F. Calegari, J. L. Sanz-Vicario, F. Martín, and M. J. J. Vrakking, Physical Review Letters 107, 043002 (2011).

[13] P. Ranitovic, C. W. Hogle, P. Rivière, A. Palacios, X.-M. Tong, N. Toshima, A. GonzálezCastrillo, L. Martin, F. Martín, M. M. Murnane, and H. Kapteyn, Proceedings of the National Academy of Sciences of the United States of America 111, 912 (2014).

[14] E. Goulielmakis, Z.-H. Loh, A. Wirth, R. Santra, N. Rohringer, V. S. Yakovlev, S. Zherebtsov, 
T. Pfeifer, A. M. Azzeer, M. F. Kling, S. R. Leone, and F. Krausz, Nature 466, 739 (2010).

[15] F. Calegari, D. Ayuso, A. Trabattoni, L. Belshaw, S. D. Camillis, S. Anumula, F. Frassetto, A. Poletto, P. Decleva, J. Greenwood, F. Martn, and M. Nisoli, Science 346, 336 (2014).

[16] S. Pabst, L. Greenman, P. J. Ho, D. A. Mazziotti, and R. Santra, Physical Review Letters 106, 053003 (2011).

[17] S. Haessler, B. Fabre, J. Higuet, J. Caillat, T. Ruchon, P. Breger, B. Carré, E. Constant, A. Maquet, E. Mével, P. Salières, R. Taïeb, and Y. Mairesse, Physical Review A 80, 011404 (2009).

[18] T. Aoto, K. Ito, Y. Hikosaka, A. Shibasaki, R. Hirayama, N. Yamamono, and E. Miyoshi, The Journal of Chemical Physics 124, 234306 (2006).

[19] P. Baltzer, M. Larsson, L. Karlsson, B. Wannberg, and M. Carlsson Göthe, Physical Review A 46, 5545 (1992).

[20] C. Van De Runstraat, F. De Heer, and T. Govers, Chemical Physics 3, 431 (1974).

[21] M. Schmidt and K. Baldridge, Journal of Computational Chemistry 14, 1347 (1993).

[22] V. Véniard, R. Taïeb, and A. Maquet, Physical Review Letters 74, 4161 (1995).

[23] J. Caillat, A. Maquet, S. Haessler, B. Fabre, T. Ruchon, P. Salières, Y. Mairesse, and R. Taïeb, Physical Review Letters 106, 093002 (2011).

[24] J. Tennyson, Physics Reports 491, 29 (2010).

[25] A. Abedi, N. Maitra, and E. Gross, Physical Review Letters 105, 1 (2010).

[26] A. G. Harvey, D. S. Brambila, F. Morales, and O. Smirnova, Journal of Physics B: Atomic, Molecular and Optical Physics 47, 215005 (2014).

[27] M. Spanner and S. Patchkovskii, Chemical Physics 414, 10 (2013). 